Supporting Information

\title{
Intranasal Delivery of Temozolomide-conjugated Gold Nanoparticles Functionalized with Anti-EphA3 for \\ Glioblastoma Targeting
}

Liangxiao Wang ${ }^{1}$, Shengnan Tang ${ }^{1}$, Yawen Yu $u^{1}$, Yanan $L v^{1}$, Aiping Wang*1, Xiuju

Yan $^{1}$, Nuannuan $\mathrm{Li}^{1}$, Chunjie Sha ${ }^{2}$, Kaoxiang Sun ${ }^{1}$, Youxin $L i^{* 1}$

${ }^{1}$ School of Pharmacy, Key Laboratory of Molecular Pharmacology and Drug

Evaluation (Yantai University), Ministry of Education, Collaborative Innovation

Center of Advanced Drug Delivery System and Biotech Drugs in Universities of

Shandong, Yantai University, Yantai 264005, P.R. China

${ }^{2}$ State Key Laboratory of Long-Acting and Targeting Drug Delivery System, Luye

Pharmaceutical Co., Ltd., Yantai 264003, P. R. China

*Corresponding author:

Aiping Wang

School of Pharmacy, Yantai University, No 30 Qingquan road, Yantai, 264005,

Shandong Province, People's Republic of China

Tel: +86-535-3946458, E-mail: wangaiping@luye.com

Youxin Li

School of Pharmacy, Yantai University, No 30 Qingquan road, Yantai, 264005,

Shandong Province, People's Republic of China

Tel: +86-535-3946187, E-mail: youxinli@luye.com 


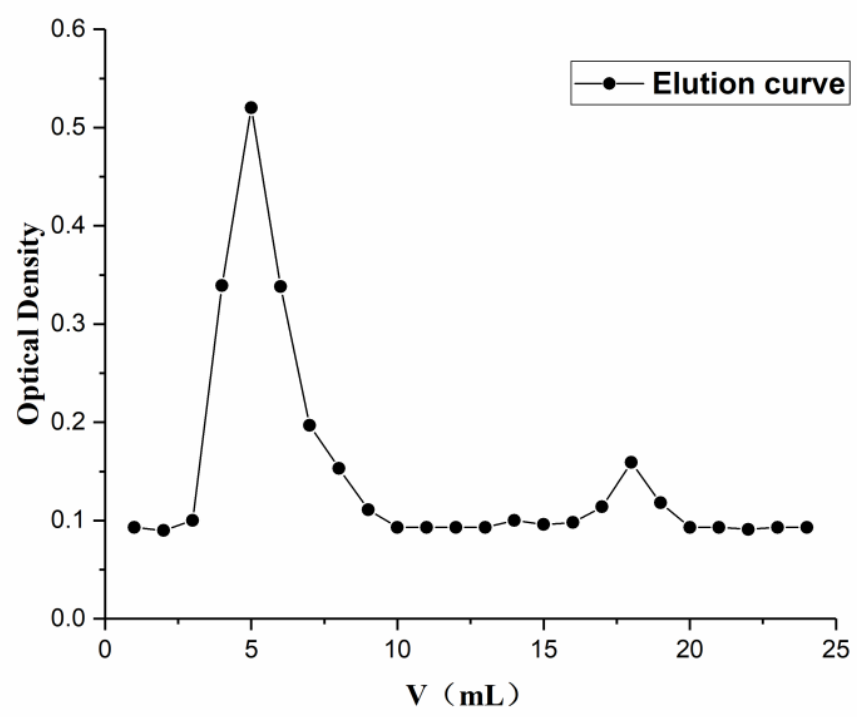

Figure S1. The elution curves of anti-EphA3-TMZ@GNPs and dissociative antiEphA3. Burgundy-red solution (3 9) with high OD value can be judged as anti-EphA3TMZ@GNPs, indicating that anti-EphA3 is connected successfully to TMZ@GNPs, the solution (17 19) is free anti-EphA3. 


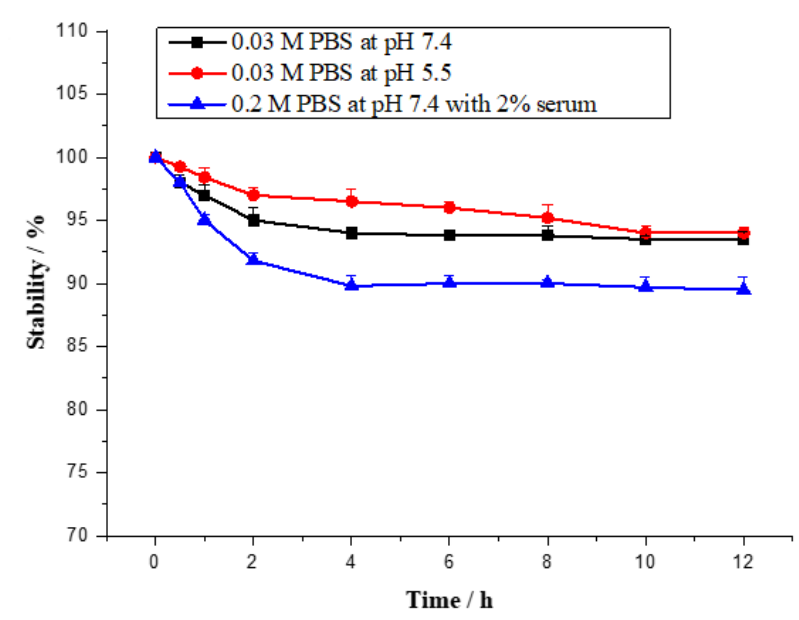

Figure S2. Dispersion stability of anti-EphA3-TMZ@GNPs in the following conditions: (1) $0.03 \mathrm{M} \mathrm{PBS}$ at $\mathrm{pH} 7.4$; (2) $0.03 \mathrm{M}$ PBS at $\mathrm{pH} 5.5$; (3) $0.2 \mathrm{M}$ PBS at $\mathrm{pH}$ 7.4 with $2 \%$ serum. Absorbance of the conjugates at $520 \mathrm{~nm}$ was monitored at $0.5,1,2$, 4, 6, 8,10 and $12 \mathrm{~h}$. $(n=3)$. The relative absorbance of each group within $12 \mathrm{~h}$ was not lower than $85.0 \%$ of anti-EphA3-TMZ@GNPs at the indicated time points, indicating that anti-EphA3-TMZ@GNPs are stable in various environments. 
Table S1. Statistics of survival time of glioma-bearing rats in each group

\begin{tabular}{lccc}
\hline Groups & Mean (days) & Median (days) & Increase of life span (\%) \\
\hline Saline & 16.6 & 17 & - \\
Free TMZ & 19.6 & 20 & 18.1 \\
TMZ @GNPs & 29.6 & 30 & 78.3 \\
anti-EphA3-TMZ@GNPs & 41.6 & 42 & 150.6 \\
\hline
\end{tabular}

